\title{
Contextual fear conditioning in zebrafish
}

\author{
Justin W. Kenney, ${ }^{1}$ Ian C. Scott, ${ }^{2}$ Sheena A. Josselyn, ${ }^{1,3,4,5}$ and Paul W. Frankland ${ }^{1,3,4,5}$ \\ ${ }^{1}$ Program in Neurosciences and Mental Health; ${ }^{2}$ Program in Development and Stem Cell Biology, The Hospital for Sick Children, Toronto, \\ Ontario, M5G 1 X8, Canada; ${ }^{3}$ Department of Physiology; ${ }^{4}$ Department of Psychology, University of Toronto, Toronto, Ontario, M5S \\ 1A8, Canada; ${ }^{5}$ Institute of Medical Sciences, University of Toronto, Toronto, Ontario, M5S 1A8, Canada
}

\begin{abstract}
Zebrafish are a genetically tractable vertebrate that hold considerable promise for elucidating the molecular basis of behavior. Although numerous recent advances have been made in the ability to precisely manipulate the zebrafish genome, much less is known about many aspects of learning and memory in adult fish. Here, we describe the development of a contextual fear conditioning paradigm using an electric shock as the aversive stimulus. We find that contextual fear conditioning is modulated by shock intensity, prevented by an established amnestic agent (MK-801), lasts at least $14 \mathrm{~d}$, and exhibits extinction. Furthermore, fish of various background strains $(A B, T u$, and $T L)$ are able to acquire fear conditioning, but differ in fear extinction rates. Taken together, we find that contextual fear conditioning in zebrafish shares many similarities with the widely used contextual fear conditioning paradigm in rodents. Combined with the amenability of genetic manipulation in zebrafish, we anticipate that our paradigm will prove to be a useful complementary system in which to examine the molecular basis of vertebrate learning and memory.
\end{abstract}

Zebrafish are a useful model organism for studying physiology due to their genetic accessibility, low cost, and potential for highthroughput analysis (Grunwald and Eisen 2002). Given that $\sim 70 \%$ of human genes have an obvious ortholog in fish (Howe et al. 2013), zebrafish can be used to model various human diseases (Lieschke and Currie 2007) and are emerging as a powerful tool for the in vivo screening of compounds for drug discovery (Zon and Peterson 2005; MacRae and Peterson 2015). More recently, both larval and adult zebrafish have been successfully used to study both basic questions in neuroscience, and gain a deeper understanding of the genetics of neuropsychiatric and neurodegenerative diseases (Agetsuma et al. 2010; Ahrens et al. 2012; Schmid and Haas 2013; Kalueff et al. 2014; Leung and Mourrain 2016).

The strength of zebrafish for understanding the genetic basis of behavior has been realized using forward genetic screens in both adult and larval fish (Darland and Dowling 2001; Muto et al. 2005; Gerlai 2010). With the advent of scalable genome editing technologies, such as clustered regularly interspaced short palindromic repeats (CRISPR)/Cas9, the potential for performing targeted highthroughput reverse genetic screens is now also feasible (Hwang et al. 2013; Varshney et al. 2015). Additionally, zebrafish hold considerable potential in personalized medicine, as the CRISPR/Cas9 system has been successfully used to precisely knock-in exogenous DNA (Hisano et al. 2015). However, to fully leverage the zebrafish model to understand how genetics contributes to behavior in both health and disease requires a deep understanding of zebrafish behavior.

Adult zebrafish exhibit a rich repertoire of behaviors, from complex social interactions, to anxiety-like behaviors, and various forms of learning (Kalueff et al. 2013; Blaser and Vira 2014; Gerlai 2015). Associative learning is an important, and highly conserved form of learning in which an initially neutral conditioned stimulus (CS) is paired with an unconditioned stimulus (US) resulting in the expression of a conditioned response (CR) upon subsequent exposure to the CS. Although both classical (Pavlovian) and operant conditioning have been demonstrated in zebrafish (Arthur and Levin 2001; Xu et al. 2007; Blank et al. 2009; Agetsuma et al. 2010; Sison and Gerlai 2010; Aizenberg and Schuman 2011;

\section{Corresponding author: paul.frankland@sickkids.ca}

Article is online at http://www.learnmem.org/cgi/doi/10.1101//m.045690.117.
Valente et al. 2012; Manuel et al. 2014; Gorissen et al. 2015; Fernandes et al. 2016), many of these tasks require habituation or training over multiple days, do not last beyond $24 \mathrm{~h}$ following training, are difficult to assess, or are not generalizable to fish of different genetic backgrounds. Here, we describe the development of a contextual fear conditioning task in adult zebrafish that is robust, rapidly acquired, straightforward to measure, and allows for the examination of the various phases of learning (acquisition, consolidation, and retrieval).

\section{Results}

\section{Shock intensity}

We initially determined an appropriate shock intensity to reliably elicit both an unconditioned response (UR) and a CR in zebrafish. We administered shocks ranging from 0 to $20 \mathrm{~mA}$ individually to fish during the training period (Fig. 1A; $0 \mathrm{~mA}: n=20,5 \mathrm{~mA}: n=$ 19, $10 \mathrm{~mA}: n=20,20 \mathrm{~mA}: n=19)$. We found that a 20 -mA shock resulted in both a robust UR (i.e., an activity burst during shock administration) and CR (i.e., a decrease in distance traveled following repeated shock administration) whereas a 10-mA shock resulted in a less consistent UR and no CR, and the 5-mA shock resulted in no discernible change in locomotor activity (Fig. 1B).

The UR of fish to the shock stimulus differed depending on the shock intensity administered $\left(F_{(3,74)}=41.5, P=7.8 \times 10^{-16}\right)$ with a Dunnett post hoc tests indicating that the 10 and $20 \mathrm{~mA}$ groups differed from the no shock group (5 mA: $P=0.88,10 \mathrm{~mA}$ : $P=0.0015,20 \mathrm{~mA}: P<0.0001$ Fig. 1C). Additionally, the change in distance traveled of fish subjected to different shock intensities differed during the last half of the training trial $\left(F_{(3,74)}=19.6, P=\right.$ $1.8 \times 10^{-9}$ ) with a Dunnett post hoc test indicating that only the 20 -mA group differing significantly from the no shock group (5 mA: $P=0.88,10 \mathrm{~mA}: P=0.93,20 \mathrm{~mA}: P<0.0001$; Fig. 1D).

When placed back into the tank during the test, all three groups administered shocks demonstrated a CR in that they

\footnotetext{
(C) 2017 Kenney et al. This article is distributed exclusively by Cold Spring Harbor Laboratory Press for the first 12 months after the full-issue publication date (see http://learnmem.cshlp.org/site/misc/terms.xhtml). After 12 months, it is available under a Creative Commons License (Attribution-NonCommercial 4.0 International), as described at http://creativecommons.org/licenses/ by-nc/4.0/.
} 
A

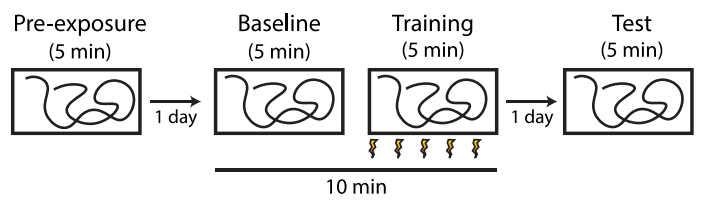

B
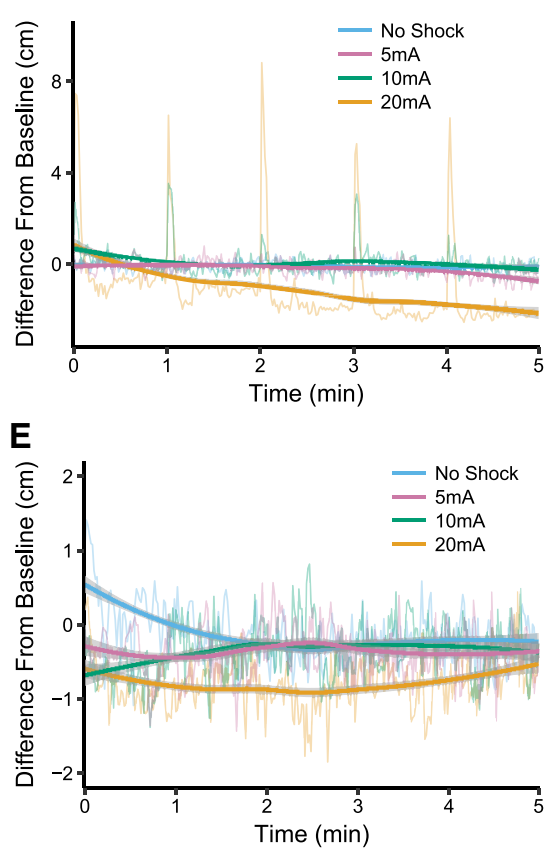

C
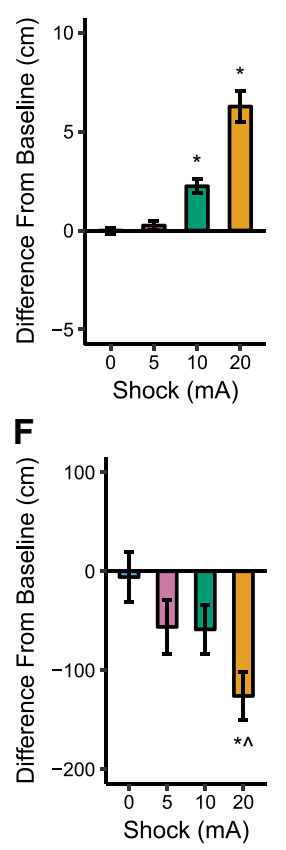

Figure 1. Contextual fear conditioning in adult zebrafish. ( $A$ ) Scheme indicating behavioral procedure used for inducing fear conditioning in zebrafish. $(B)$ Second-by-second differences from baseline in distance traveled during training in contextual fear conditioning. $(C)$ Differences from baseline in distance traveled during shock administration at training. (D) Differences from baseline in distance traveled over the last $2.5 \mathrm{~min}$ of training. (E) Second-by-second differences from baseline in distance traveled during the 5-min test session. $(F)$ Differences from baseline in distance traveled over the first 2.5 min of testing. $(G)$ Differences from baseline in distance traveled over the first $2.5 \mathrm{~min}$ of testing for individually housed fish. Semitransparent lines are averaged second-by-second data. Solid lines are the result of a local polynomial regression fit with $95 \%$ confidence interval for the fit (gray ribbons). $\left({ }^{*}\right) P<0.05$ compared with unshocked fish, $\left({ }^{\wedge}\right) P<0.05$ compared with a difference score of zero, $n=19-20(A-F), n^{\prime} s=10-12(G)$.

initially had a decrease in their locomotor activity; however, the 5 and $10 \mathrm{~mA}$ groups returned to baseline levels of activity within 2 min, whereas the decrease in swimming in the $20 \mathrm{~mA}$ group was persistent throughout the trial (Fig. 1E). Examination of the first half of the testing trial confirmed that there was a stimulusdependent effect of treatment on distance traveled $\left(F_{(3,74)}=3.7\right.$, $P=0.015)$ with a Dunnett post hoc test indicating that only the 20-mA group differed from the no shock group (5 mA: $P=0.37$, $10 \mathrm{~mA}: P=0.32,20 \mathrm{~mA}: P=0.0039$; Fig. 1F). Furthermore, Bonferroni corrected one-sample $t$-tests found a difference from zero for the $20 \mathrm{~mA}$ group, but not the 5 or $10 \mathrm{~mA}$ groups (no shock: $P=1,5 \mathrm{~mA}: P=0.22,10 \mathrm{~mA}: P=0.12,20 \mathrm{~mA}: P=0.00028$ ).

Because all fish in a single experimental group were housed together (but trained and tested individually), and fish are known to release diffusible pheromones into the water that could affect the behavior of tankmates, we examined fear conditioning in fish that were individually housed ( $0 \mathrm{~mA}: n=13,20 \mathrm{~mA}: n=12)$. We found that a $20-\mathrm{mA}$ shock was also effective at inducing fear conditioning in singly housed fish $\left(t_{(20)}=5.54, P=2.0 \times 10^{-5}\right.$; Fig. $1 \mathrm{G})$. This suggests that the fear conditioning we observe in group housed fish is unlikely to be affected by the behavior or pheromone release of conspecifics.

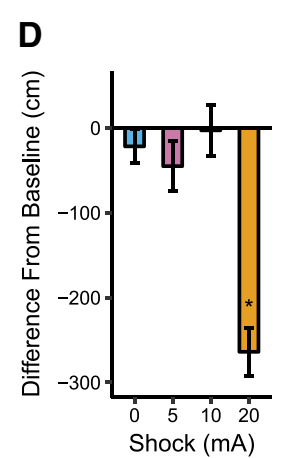

G

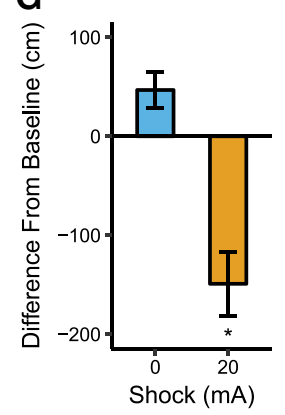

Taken together, we find that administration of $20-\mathrm{mA}$ shocks results in the formation of a robust CS-US (tank-shock) association with less pronounced effects using the 5 and $10 \mathrm{~mA}$ shock intensities. Furthermore, the effect on behavior during testing appears to be most pronounced during the first half of the testing trial (Fig. 1E) and is present in both group and individually housed fish. Based on these findings, we used the 20-mA shock intensity throughout the rest of our experiments and focus our analysis on the first $2.5 \mathrm{~min}$ of testing.

\section{Tank specificity}

The decrease in swimming we observed following shock administration to zebrafish may reflect injury, and not a conditioned fear response. Therefore, we sought to determine whether fear conditioning in zebrafish is context-specific, a well-known characteristic of contextual fear conditioning in rodents (Owen et al. 1997; Rudy and O'Reilly 1999). To accomplish this, we interspersed exposure to another novel tank (tank B) with exposure to the tank in which shocks were administered (tank A; Fig. 2A; $n=$ 30). When fish were tested in tank A, they had a persistent decrease in their distance traveled relative to baseline compared with testing in tank B (Fig. 2B). Comparing fish during the first half of the trial confirmed that testing in tank A resulted in a decrease in locomotor activity compared with testing in tank B $\left(t_{(58)}\right.$ $=2.82, P=0.0066$ ), with Bonferroni corrected one sample $t$-tests indicating that the difference in swimming from baseline was significantly different from zero in Tank A $\left(P=1.16 \times 10^{-5}\right)$, but not tank B $(P=0.53$; Fig. 2C). These data suggest that zebrafish are able to discriminate between tanks, and thus the conditioned response is unlikely to be due to injury.

\section{MK-801}

Learning in a variety of species, including zebrafish, is known to be sensitive to amnestic drugs such as MK-801, a noncompetitive NMDA receptor antagonist (Abel and Lattal 2001; Blank et al. 2009; Sison and Gerlai 2011a). To determine whether the consolidation of contextual fear conditioning is sensitive to a known amnestic agent, we exposed zebrafish to $20 \mu \mathrm{M}$ MK-801 following training in contextual fear conditioning (Fig. 3; vehicle: $n=18$; MK-801: $n=19)$. MK-801 was administered following acquisition of fear conditioning as it has been reported to alter baseline locomotion in both zebrafish and mice (Swain et al. 2004; Roberts et al. 2011; but see Clineschmidt et al. 1982; Wu et al. 2005; Sison and Gerlai 2011b), which could affect assessment of learning in our paradigm. We found that fish treated with vehicle had a persistent decrease in locomotor activity during testing, whereas those treated with MK-801 did not (Fig. 3A). An examination of the first half of testing confirmed that vehicle-treated fish suppressed their locomotor activity compared with MK-801-treated fish $\left(t_{(35)}=3.30, P\right.$ 
A

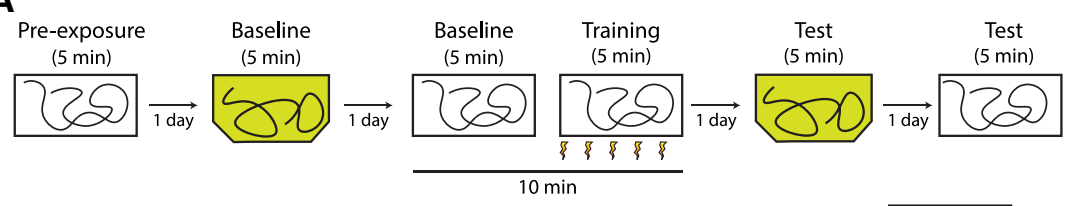

B
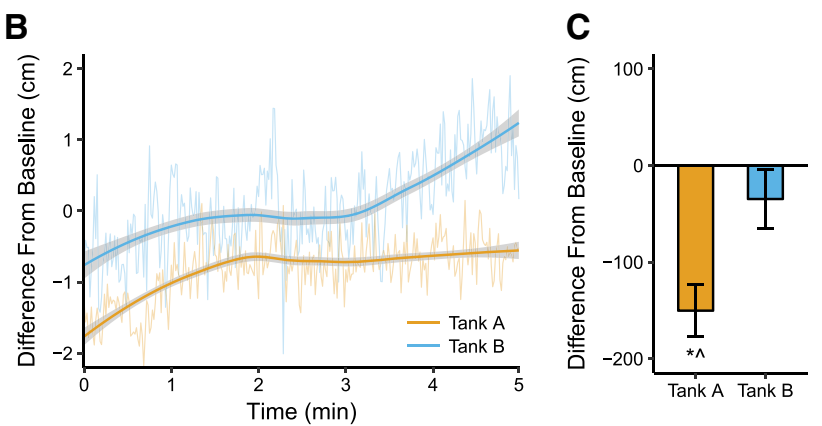

Figure 2. Contextual (tank) discrimination in adult zebrafish. (A) Scheme indicating the behavioral procedure used to test for tank discrimination. $(B)$ Averaged second-by-second differences from baseline in distance traveled during testing in contextual fear conditioning in either tank A or tank B. (C) Differences from baseline in distance traveled over the first $2.5 \mathrm{~min}$ of the test session. Semitransparent lines are average second-by-second data. Solid lines are the result of a local polynomial regression fit with $95 \%$ confidence interval for the fit (gray ribbons). ( $\left.{ }^{*}\right) P<0.05$ compared with testing in Tank $B,\left({ }^{\wedge}\right) P<0.05$ compared with a difference of zero, $n^{\prime} s=30$.

$=0.0022$; Fig. 3B). Using the tank specificity paradigm (Fig. 2A), we found that MK-801 administration following training had no effect on swimming activity in tank B whereas vehicle-treated fish swam less in tank A compared with MK-801-treated fish in tank A or vehicle-treated fish in tank B (group by tank interaction: $F_{(64,1)}=$ 7.51, $P=0.0079$; Bonferroni corrected post hoc tests: vehicle + tank A compared with MK-801 + tank A: $P=0.009$, vehicle + tank B: $P=0.015$. MK-801 + tank B compared with MK-801 $+\operatorname{tank}$ A, $P$ $=1$; vehicle $+\operatorname{tank} B, P=1$; Fig. 3C; vehicle: $n=18$, MK-801: $n=16$ ).

\section{Time course}

Thus far we have found that contextual fear memories are present 1 $\mathrm{d}$ following training, which is a time frame consistent with numerous previous studies examining memory in zebrafish (Blaser and Vira 2014; Kalueff et al. 2014; Gerlai 2015). However, it is largely unknown how long memories in zebrafish may last. In order to determine how long-lasting the contextual fear conditioning memory is, we trained separate groups of zebrafish with delays of $7,14,21$, or $28 \mathrm{~d}$ between training and testing (Fig. $4 ; 7 \mathrm{~d}$ : no shock: $n=19$, shock: $n=19.14$ d: no shock: $n=20$, shock: $n=20.21$ d: no shock: $n=19$, shock: $n=20.28$ d: no shock: $n=21$, shock: $n=20$ ). We found that at all delays, the decrease in locomotor activity at the beginning of testing was present (Fig. 4A-D, left). Analysis of the first half of the testing trials indicated that a robust fear memory lasts between 14 and $21 \mathrm{~d}\left(7 \mathrm{~d}: t_{(36)}=3.97, P=0.00033 ; 14 \mathrm{~d}\right.$ : $t_{(38)}=3.20, P=0.0028 ; 21 \mathrm{~d}: t_{(37)}=1.87, P=0.070 ; 28 \mathrm{~d}: t_{(39)}=$ $1.43, P=0.16$; Fig. 4 A-D, right).

\section{Extinction}

One phenomenon related to associative learning is extinction. In extinction, repeated exposure to the CS in the absence of the US results in a reduction of the CR. We attempted to extinguish the tank-shock association by repeated exposure of the fish to the conditioning tank on successive days. We found that, although within session extinction is present during each of the $4 \mathrm{~d}$ of testing, the decrease in locomotor activity at the beginning of the session persisted throughout the days of testing (Fig. 5A; no shock: $n=31$,
Tank A

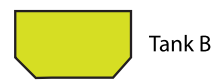

shock: $n=32$ ). A $2 \times 4$ (group $\times$ day) permutation $F$-test applied to the change in distance traveled during the first half of each testing trial found a main effect of group $(P<0.001)$, but not day $(P=0.15)$, and a day by group interaction $(P=$ 0.003; Fig. 5B). Bonferroni corrected post hoc tests found that shocked fish differed from unshocked fish at the first and second day (day $1: P=1.10 \times 10^{-7}$, day 2 : $P=0.0055$, day 3: $P=0.077$, day $4: P=$ 0.17 ), and that the shocked fish at days 3 and 4 , but not day 2, differed from shocked fish at day 1 (compared with day 1 , day 2: $P=0.47$, day $3: P=0.020$, day 4: $P=0.015)$. Taken together, these data suggest that the memory largely extinguished after four consecutive days of exposure to the conditioning tank.

\section{Tu and TL fish}

Many learning tasks in rodents, and some in fish, are known to be affected by the genetic background of the strain under study (Owen et al. 1997; Gerlai 1998; Vignet et al. 2013; Gorissen et al. 2015). Thus, we sought to determine if different widely used strains of zebrafish exhibit responses in our contextual fear conditioning paradigm that are similar to the $\mathrm{AB}$ strain we
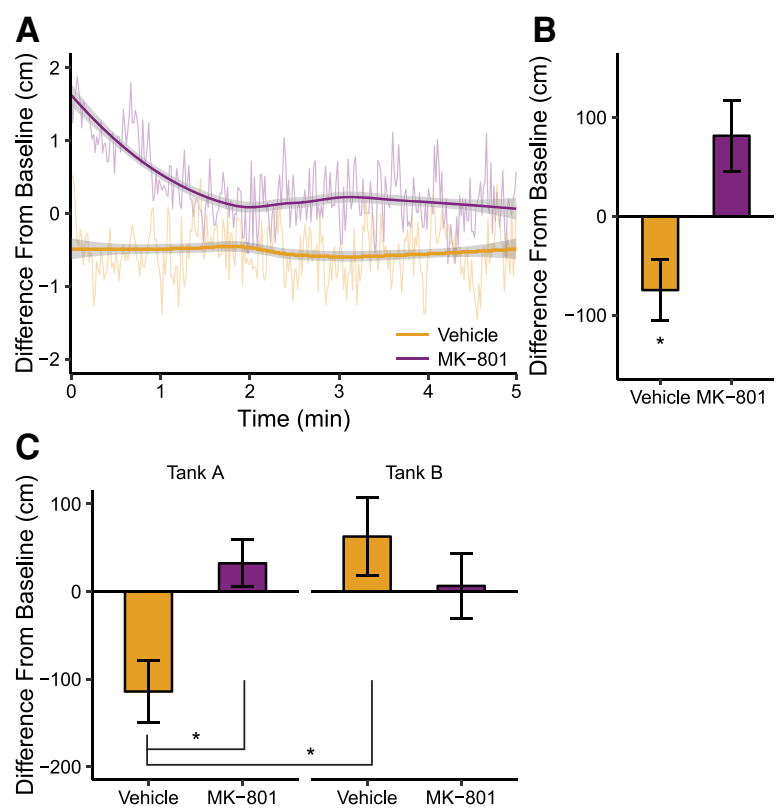

Figure 3. The effect of $20 \mu \mathrm{M}$ MK-801 administration after training on contextual fear conditioning. (A) Averaged second-by-second differences from baseline in distance traveled during testing in contextual fear conditioning in fish administered vehicle or MK-801. (B) Differences from baseline in distance traveled over the first $2.5 \mathrm{~min}$ of testing of fear conditioning. (C) Differences from baseline in distance traveled over the first $2.5 \mathrm{~min}$ of testing for fish administered vehicle or MK-801 after training and exposed to the tank specificity paradigm (Fig. 2A). Semitransparent lines are average second-by-second data. Solid lines are the result of a local polynomial regression fit with $95 \%$ confidence interval for the fit (gray ribbons). ( $\left.{ }^{*}\right) P<0.05$ compared with MK-801-treated fish or as indicated, $n^{\prime} s=18-19(A, B), n^{\prime} s=16-18(C)$. 
A

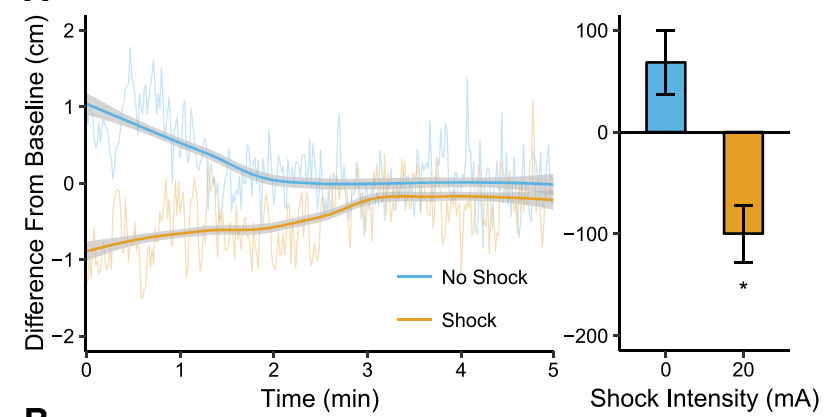

B
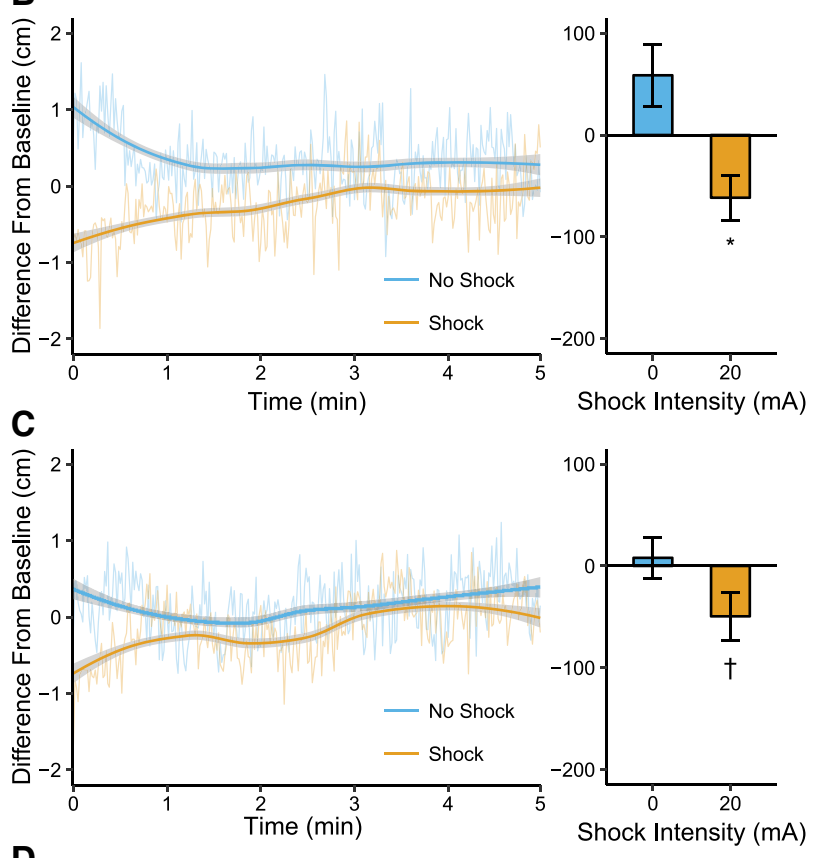

D

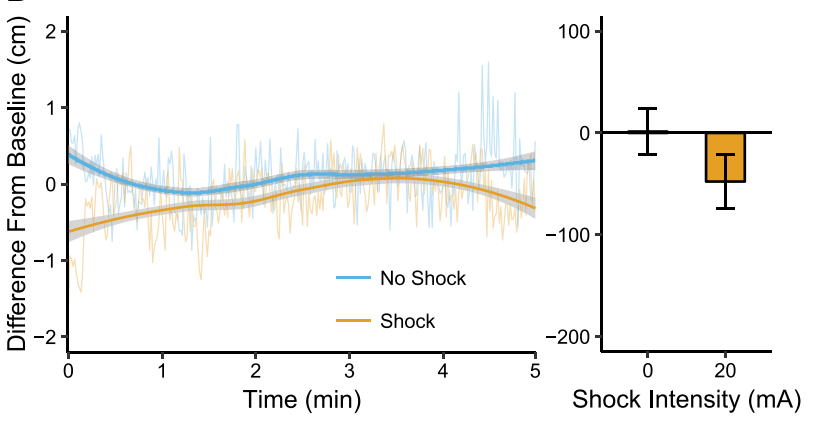

Figure 4. Effect of different retention delays on contextual fear conditioning in adult zebrafish. Fish were tested after a delay of $7(A), 14(B)$, $21(C)$, or $28(D)$ days following training. Second-by-second differences from baseline in distance traveled during the entire test (left) and during the first $2.5 \mathrm{~min}$ of testing (right). Semitransparent lines are average second-by-second data. Solid lines are the result of a local polynomial regression fit with $95 \%$ confidence interval for the fit (gray ribbons). $\left(^{*}\right) P<$ $0.05,(\dagger) P<0.10$ compared with unshocked fish, $n^{\prime} s=19-21$.

have examined thus far. Tu fish have been used for sequencing of the zebrafish genome (Howe et al. 2013) and TL fish are another commonly used strain that are genetically distinct from both the $\mathrm{AB}$ and Tu strains (Trevarrow and Robison 2004; Guryev et al. 2006). Fish of both the Tu and TL background exhibited responses during training that were similar to that of $\mathrm{AB}$ fish, although the decrease in swimming in Tu fish appeared to plateau more quickly than in either the $\mathrm{AB}$ or TL strains (Fig. $6 \mathrm{~A}, \mathrm{~B}$, left; Tu: no shock: $n=$ 15, shock: $n=17$. TL: no shock: $n=18$, shock: $n=20$ ). Both Tu and TL fish increased their locomotor activity in response to the 20-mA shock (Fig. 6A,B, right; Tu: $t_{(30)}=5.90, P=1.86 \times 10^{-6}$; TL: $t_{(36)}=$ $\left.5.19, P=8.31 \times 10^{-6}\right)$. An examination of data during successive exposures to the testing tank indicated that Tu fish had both robust within and between session extinction (Fig. 6C) whereas TL fish showed little within session extinction and only moderate between session extinction (Fig. 6D).

In Tu fish, a $2 \times 3$ (group $\times$ day) permutation $F$-test performed on the difference from baseline in distance traveled during the first half of testing during each extinction day found a main effect of group $(P<0.001)$, a main effect of day $(P=0.028)$ but no interaction between day and group $(P=0.20$; Fig. $6 \mathrm{E})$. Bonferroni corrected post hoc tests indicated that shocked Tu fish differed from unshocked fish at days 1 and 2, with a trend toward a difference on day 3 of extinction (day 1: $P=0.0024$, day 2: $P=0.0060$, day 3: $P=0.094)$. Furthermore, comparisons with the first day of testing indicated that fish significantly decreased their activity on day 3, but not day 2 , of extinction testing (compared with day 1 , day 2 : $P=0.40$, day 3: $P=0.043)$.

In TL fish, a $2 \times 4$ (group $\times$ day) permutation $F$-test using the difference from baseline in distance traveled from the first half of testing on each day found a main effect of group $(P<0.001)$, and a trend toward an effect of day $(P=0.097)$, but no group by day interaction $(P=0.28$; Fig. $6 \mathrm{~F})$. Bonferroni corrected post hoc tests comparing shocked and unshocked fish for each day found that shocked fish differed from unshocked fish at each day (day 1: $P=$

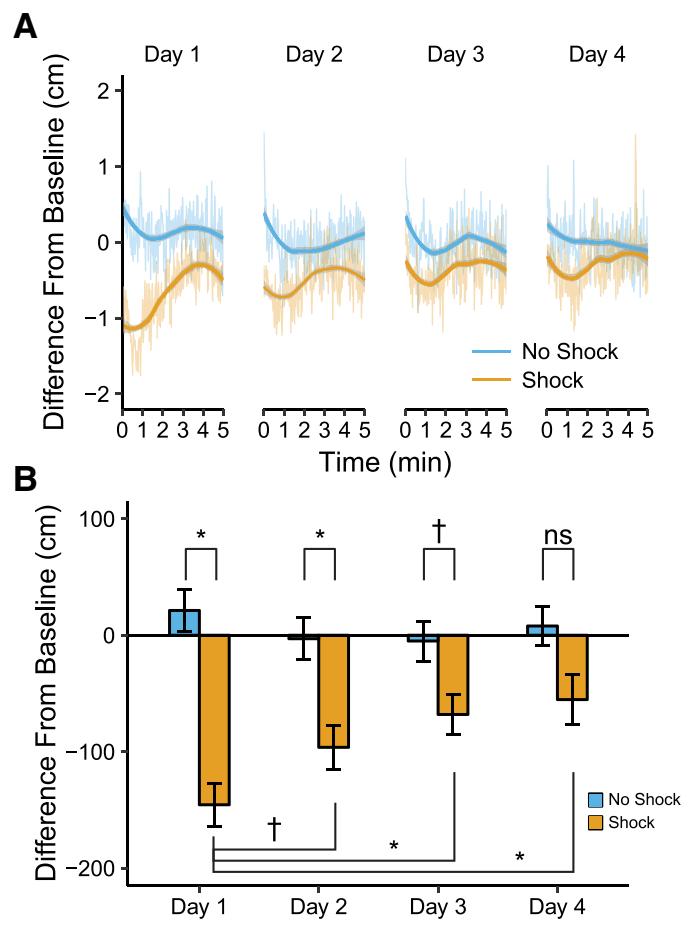

Figure 5. Extinction of contextual fear conditioning in adult zebrafish. (A) Second-by-second differences from baseline in distance traveled during each of four successive days of testing following training in contextual fear conditioning. (B) Differences from baseline in distance traveled over the first $2.5 \mathrm{~min}$ of four successive days of testing following training in contextual fear conditioning. Semitransparent lines are average second-by-second data. Solid lines are the result of a local polynomial regression fit with $95 \%$ confidence interval for the fit (gray ribbons). $\left({ }^{*}\right) P<$ $0.05,(\dagger) P<0.10, n^{\prime} s=31-32$. 
A
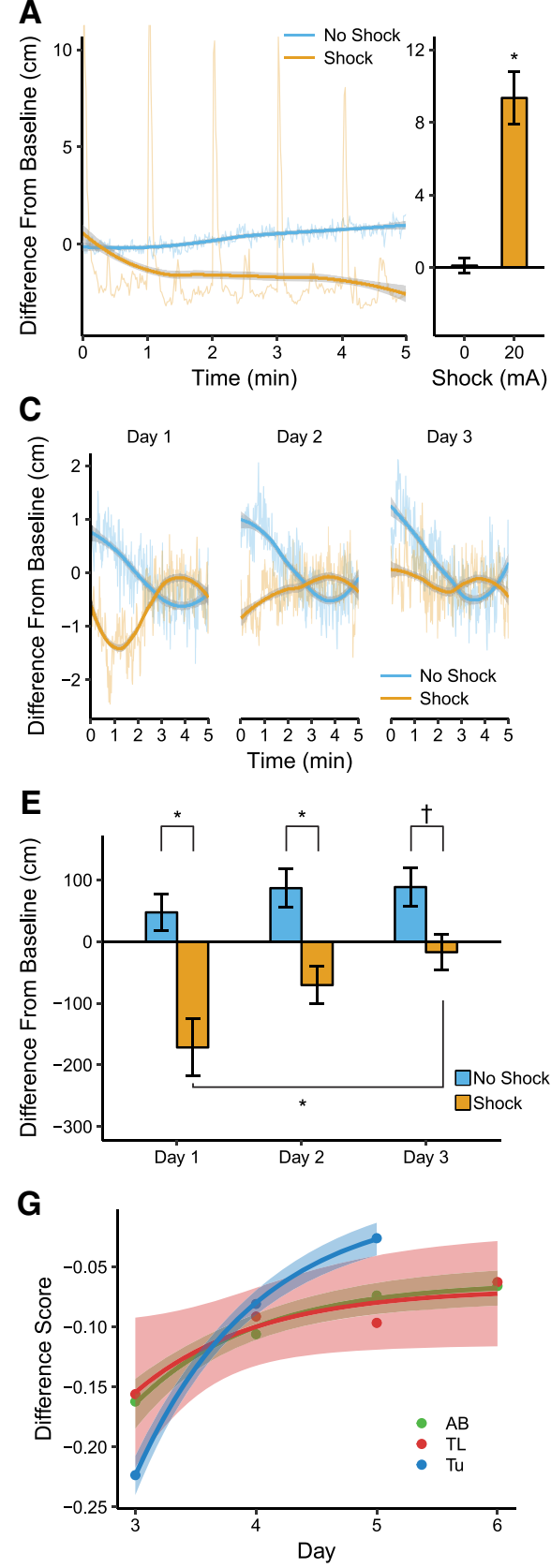

B
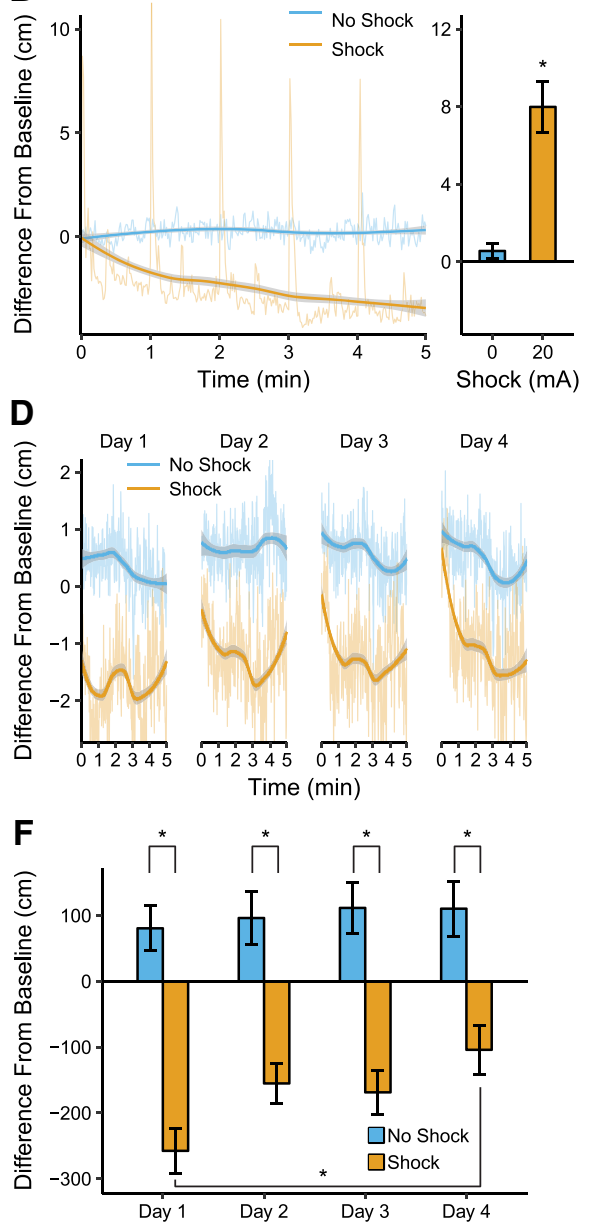

H

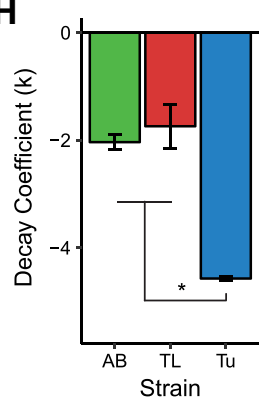

Figure 6. Contextual fear conditioning in Tu and TL zebrafish strains. Behavior during training in Tu $(A)$ and TL $(B)$ fish with second-by-second data on the left and response to the shock on the right. Second-by-second differences from baseline in distance traveled during successive days of testing in $\mathrm{Tu}(C)$ and TL $(D)$ fish. Differences from baseline in distance traveled during the first $2.5 \mathrm{~min}$ of three or four successive days of testing in Tu $(E)$ and TL $(F)$ fish. Semitransparent lines are average second-by-second data. Solid lines are the result of a local polynomial regression fit with $95 \%$ confidence interval for the fit (gray ribbons). (G) Extinction curves for AB, Tu, and TL fish fit difference scores with an exponential decay function with $95 \%$ confidence intervals for the fit. $(F)$ Decay coefficients and SEM for $A B, T u$, and TL fish derived from fitting extinction curves with an exponential decay function. $\left(^{*}\right) P<$ 0.05, (†) $P<0.10, n^{\prime} s=15-20$.

$2.10 \times 10^{-7}$, day 2: $P=0.00013$, day 3: $P=2.54 \times 10^{-5}$, day $4: P=$ $0.0037)$ and that in shocked fish only testing on day 4 was different from the first day of testing (compared with day 1 , day $2: P=0.20$, day 3: $P=0.49$, day $4: P=0.030)$.

To determine whether the $\mathrm{AB}, \mathrm{Tu}$, and TL strains of fish differ with respect to their rate of fear memory extinction, we fit expo- nential decay curves to average difference scores across days for each strain (Fig. 6G). We found the decay coefficient $(k)$ to be significantly larger for Tu fish as compared with $\mathrm{AB}$ or TL fish (AB: $k=-2.04$, 95\% CI $[-2.62,-1.45]$; Tu: $k=-4.57$, $95 \%$ CI $[-5.10,-4.04]$; TL: $k=-1.74$, 95\% CI $[-3.49,0.0099]$; Fig. $6 \mathrm{H})$. This suggests that the contextual fear memory in Tu fish extinguishes more quickly than in $\mathrm{AB}$ or TL fish.

We used our tank specificity paradigm (Fig. 2A) to determine whether or not Tu and TL fish demonstrated a specific CR during testing in fear conditioning, and were unlikely to be injured during training (Tu: $n=36$, TL: $n=33$ ). We found that Tu fish had a decrease in swimming relative to baseline when placed back in tank A versus tank B $\left(t_{(70)}=4.66, P=\right.$ $1.46 \times 10^{-5}$ ) and the difference from baseline in $\operatorname{tank} A$, but not tank B, was significantly different from zero (Bonferroni corrected, tank A: $P=7.95 \times 10^{-6}$, tank B: $P=0.31$; Fig. 7A,B). Similarly, there was a decrease in swimming in TL fish in tank A versus tank B $\left(t_{(64)}=2.15, P=0.035\right)$ and the difference from baseline in tank A, but not tank B, was significantly different from zero (Bonferroni corrected, tank A: $P=0.00034$; $\operatorname{tank} B: P=0.85$; Fig. $7 \mathrm{C}$, D). This suggests that, like $\mathrm{AB}$ fish, both Tu and TL fish can differentiate between tanks A and B, and are unlikely to have been injured by the shock.

\section{Discussion}

In the present study, we find that contextual fear conditioning in zebrafish is a robust and rapidly acquired task that allows for the examination of numerous types of learning. Contextual fear conditioning can be modulated by the strength of the shock, lasts at least $14 \mathrm{~d}$, is sensitive to an amnestic agent (MK-801), extinguishes following repeated exposure to the context (tank), and can be acquired by fish with a variety of genetic backgrounds. Furthermore, the apparatus was built from inexpensive off-the-shelf components and the analyses were performed using open-source software, thereby making this behavioral paradigm easily scalable and widely accessible. However, some weaknesses of the current experimental design are that fish identities were not tracked across days, and all fish in the same tank were in the same experimental group. These shortcomings can be ameliorated in future work by making use of methods to tag and track individual fish housed in groups (Hohn and Petrie-Hanson 2013).

The simplest alternative explanation for the findings in the present study is that the decrease in locomotor activity observed following shock administration is due to injury and not the 
A
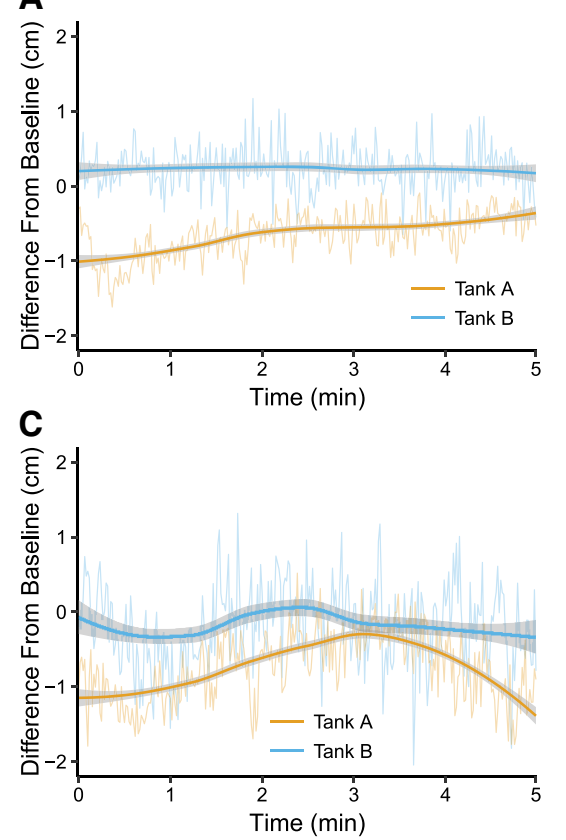

B
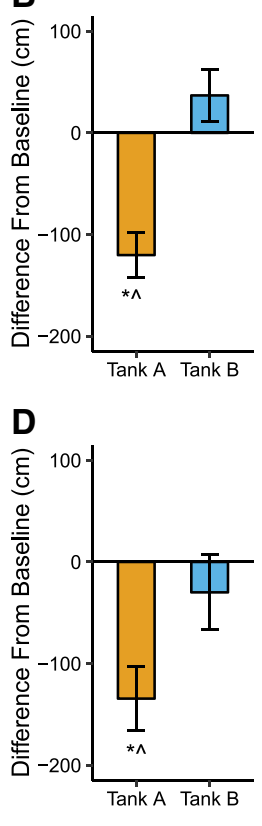

Figure 7. Contextual (tank) discrimination in Tu and TL zebrafish. Behavior during testing in Tu $(A, B)$ and TL $(C, D)$ fish in Tank A or Tank $B$ with second-by-second differences from baseline (left) and differences from baseline over the $2.5 \mathrm{~min}$ of the test session (right) in distance traveled. Semitransparent lines are average second-by-second data. Solid lines are the result of a local polynomial regression fit with $95 \%$ confidence interval for the fit (gray ribbons). $\left.{ }^{*}\right) P<0.05$, compared with testing in Tank $B,(\wedge) P<0.05$ compared with a difference of zero, $n^{\prime} s=33-36$.

expression of a CR arising from the formation of a tank-shock association. However, several aspects of our findings argue against this interpretation. First, in almost every experiment, there is significant within session extinction where the decrease in locomotor activity is greatest during the first half of the test session and approaches zero during the second half of testing. If the fish were injured by the shock, we would expect the decrease in locomotor activity to remain constant throughout the session. Second, if fish were injured due to the shock, it is unlikely that administration of MK-801 would be able to prevent learning in this paradigm (Fig. 3 ). Third, fish are able to modulate their decrease in locomotor activity based on the tank in which they are placed where the decrease is greater in the tank they were shocked in as opposed to a different novel tank (Figs. 2, 3C, 7). If the suppression of locomotor activity were due to injury, we would expect the decrease to be the same in both tanks. Finally, we see no effect on locomotor activity after placement back in home tanks immediately following shock administration when any such effect of injury would be most obvious (JW Kenney, unpublished observation).

Although a number of other aversive learning tasks have been described in zebrafish, none have been reported to be as rapidly acquired, robust, and generalizable as the contextual fear conditioning task we describe here. For example, inhibitory avoidance in zebrafish has been found to be rapidly acquired, requiring only one trial for learning (Blank et al. 2009). However, some strains of fish, such as the widely used $A B$ strain, are unable to learn inhibitory avoidance (Gorissen et al. 2015) whereas we find that the $A B$, $\mathrm{Tu}$, and TL strains all demonstrate robust learning of contextual fear conditioning. Both operant and classical fear conditioning to a discrete visual cue have been described in adult zebrafish using a shock (Agetsuma et al. 2010; Valente et al. 2012) or alarm phero- mone as the US (Hall and Suboski 1995). However, work using shocks required multiple trials with the memory lasting no longer than $6 \mathrm{~h}$ (Agetsuma et al. 2010; Valente et al. 2012), and the alarm pheromone only elicits an unconditioned response in $\sim 40 \%$ of fish (Hall and Suboski 1995). In contrast, we find that our contextual fear conditioning memory lasts at least $14 \mathrm{~d}$ (Fig. 4) and the shock US elicits a measurable, robust, and consistent increase in locomotor activity across several strains of fish (Figs. 1, 6).

To the best of our knowledge, the present study is the first demonstration of memory retention beyond $24 \mathrm{~h}$ after one-trial learning in zebrafish. Establishment of a learning task with a clearly delineated acquisition phase and sufficient strength to last weeks is useful as it allows for the study of not only molecular/cellular consolidation that occurs over $24 \mathrm{~h}$ following learning, but also slower processes (e.g., systems consolidation or forgetting) that may emerge over the course of days and weeks rather than hours (Abel and Lattal 2001; Wixted 2004; Frankland and Bontempi 2005; Frankland et al. 2013). Interestingly, we found that in zebrafish the strength of the fear memory decreased progressively as the interval between training and testing increased, suggesting natural forgetting occurred across this period (Fig. 4). This is in contrast to what is observed in rodents, where fear memories tend to have the same or greater strength at increasing delays (Fanselow et al. 1994; Houston et al. 1999).

In addition to contextual fear learning, our learning paradigm can also be used to study extinction. Extinction of associative memories has previously been reported in zebrafish for inhibitory avoidance (Piato et al. 2011), visual discrimination learning (Colwill et al. 2005), and conditioned place avoidance (Yu et al. 2006). Here, we find that extinction often occurs both within a single testing session as well as between sessions during repeated exposure to the testing tank. Interestingly, we find that fish of the $\mathrm{Tu}$ background extinguish more quickly than fish from the $\mathrm{AB}$ and TL genetic backgrounds. This is similar to what is observed in the extinction of contextual fear in rodents, where the extinction of the fear response has been observed to differ between strains of rodents (Stiedl et al. 1999; Camp et al. 2009).

Taken together, we find contextual fear conditioning in adult zebrafish to be a reliable, long-lasting, and versatile task for studying various aspects of associative learning. The paradigm described here is similar in many ways to contextual fear conditioning in rodents that has proven to be a powerful tool for probing the many facets of learning and memory (Johansen et al. 2011; Maren et al. 2013). The robustness of fear conditioning, along with the ability to modulate the strength of learning via shock intensity, makes it suitable to examine factors that may either enhance or inhibit learning.

\section{Materials and Methods}

\section{Subjects}

Subjects were $\mathrm{AB}, \mathrm{Tu}$, or TL zebrafish 3-12 mo of age. Male and female fish were housed together, 7-11 per $2 \mathrm{~L}$ tank (with the exception of when fish were individually housed; Fig. 1G). All fish were bred and raised at the Hospital for Sick Children in high-density racks under standard conditions with a 14:10 light-dark cycle (lights on at 8:30). Fish were fed twice daily with Artemia salina. Behavioral testing took place between 12:00 and 18:00. All procedures were approved by the Hospital for Sick Children Animal Care and Use Committee.

\section{Apparatus}

The behavioral apparatus consisted of two identical Aquaneering crossing tanks (ZHCT100T; $19.8 \times 8.9 \times 7.3 \mathrm{~cm}$ ) covered with white self-adhesive film and two horizontal black stripes to provide visual cues. Two stainless steel mesh grids were also placed at each end of 
the tank that served as electrodes. Tanks were surrounded by white Plasticore to prevent interference from external visual stimuli. Webcams (Logitech C270) were mounted $\sim 35 \mathrm{~cm}$ above tanks. An alternate tank (tank B) consisted of a $1.8 \mathrm{~L}$ Aquaneering tank (ZT180) with blue and green stripes on the bottom. To further differentiate the alternate tank from the training tank, several visual stimuli (photographs of Norwegian Fjords) were placed on the inside walls of the Plasticore enclosure. A Nextech (c-215) camera was placed $\sim 40 \mathrm{~cm}$ in front of the tank. Videos were recorded on a laptop PC using Free Screencast software at $\sim 15$ frames per second and encoded using the Xvid MPEG-4 codec. DC electric shocks were administered using a Bio-Rad PowerPac Basic power supply connected to stainless steel mesh electrodes.

\section{Behavioral procedure}

The basic behavioral procedure had three phases (Fig. 1A): preexposure, baseline/training, and testing. During preexposure on day 1 , fish were placed individually in the test tank for $5 \mathrm{~min}$. On day 2, baseline and training, fish were placed individually in the test tank for $10 \mathrm{~min}$. Locomotor activity during the first half of the session served as baseline. During the second half of the session, fish received five shocks (3 sec, 5-20 mA) spaced 1-min apart starting 5 min into the session. On day 3 , fish were individually placed back in the test tank for 5 min.

Tank specificity experiments made use of a modified procedure that took place over $5 \mathrm{~d}$ (Fig. 2A): On days 1 and 2, fish were exposed to the conditioning tank (tank A) or alternative tank (tank B) for $5 \mathrm{~min}$, respectively. On day 3 baseline and training were conducted in tank A as described above using 20-mA shocks. On days 4 and 5 , fish were placed back into tanks B and A for 5 min each, respectively.

Video was recorded for each session and saved for offline analysis. Before and after each individual fish was placed in the apparatus, tanks were rinsed with distilled water and filled with $800 \mathrm{~mL}$ or $1.8 \mathrm{~mL}$ of fresh system water for tanks A or B, respectively (conductance of $\sim 810-820 \mu \mathrm{S} / \mathrm{cm}$ ). Clear plastic lids were placed on the top of tank A during recording. At least two separate tanks of fish were used for each experimental group.

\section{Behavioral analysis}

Videos of individual fish were tracked using Ctrax (Branson et al. 2009) and the distance traveled during each second was calculated using a custom written Python script (available at http://github. com/jkenney9a/Zebrafish). The difference between baseline and testing was used to quantify the change in swimming activity during a given test period compared with the baseline period:

$$
\text { Difference }_{i, \text { test }(t)}=D_{i, \text { test }(t)}-\overline{D_{T(i) \text {,baseline }(t)}}
$$

where $D$ is distance traveled, $i$ is an individual fish, $t$ is time, and $T$ (i) is the tank that fish $i$ is from. Because the fish were group housed and we could not reliably distinguish individual fish from one another, the average distance traveled for a given tank was used as the baseline measure $(\bar{D})$ except for the experiment in which fish were individually housed. When calculating the difference score during training, the following equation was used:

$$
\text { Difference }_{i, \operatorname{train}(t)}=D_{i, \operatorname{train}(t)}-\overline{D_{i, \text { baseline(240 } \leq t \leq 299)}}
$$

where the baseline distance traveled was calculated as the average distance the individual fish traveled during each second of the last minute of baseline.

Data from training and testing in fear conditioning are presented in two formats: (1) Average differences for each second of training or testing calculated as described above. Given the considerable noise in second-by-second data, these data are fit using a local polynomial regression to provide a measure of the overall trend in the data. (2) An average of the difference in swimming during the first or second half ( $2.5 \mathrm{~min}$ ) of training or testing. In the experiment in which fish were individually housed (Fig. 1G), if fish swam $<50 \mathrm{~cm}$ during baseline they were removed from analysis (one fish). We used data generated from this second method for inferential statistics to determine group differences and are presented as the mean \pm SEM.

\section{Drug administration}

A stock solution of MK-801 (0924; Tocris) was diluted into $100 \mathrm{~mL}$ of facility water. Fish were placed in a $250 \mathrm{~mL}$ semiopaque beaker with MK-801 $(20 \mu \mathrm{M})$ or vehicle (water) immediately following training in fear conditioning for $15 \mathrm{~min}$. The dose of MK-801 was chosen based on previous studies in adult zebrafish finding that this dose interferes with memory formation with minimal effects on locomotor activity (Blank et al. 2009; Sison and Gerlai 2011a,b).

\section{Statistical analyses}

Data were analyzed with one-way ANOVAs or permutation $F$-tests as appropriate. Permutation $F$-tests were performed for extinction experiments by random resampling of data without replacement 1000 times while shuffling day and group assignment. Dunnett post hoc tests were used to compare multiple groups to unshocked fish as indicated. Experiments with two groups were analyzed using independent samples $t$-tests. When multiple pair-wise comparisons were made using one-sample or independent samples $t$-tests, results were corrected using the Bonferroni method as indicated.

For the comparison of extinction data across fish strains, differences in swimming distances were put on the same scale using the difference score:

$$
\mathrm{DS}_{i, \text { test }(T)}=\frac{D_{i, \text { test }(t)}-\overline{D_{T(i), \text { baseline }(t)}}}{D_{i, \text { test }(t)}+\overline{D_{T(i), \text { baseline }(t)}}}
$$

where DS is the difference score. The extinction data were then fit using an exponential decay model $\left(y=A e^{-k t}\right)$ where $A$ is the $y$-intercept and $k$ is the decay rate. Ninety-five percent confidence intervals from decay coefficient $(k)$ fits were used for statistical inference. All statistical analyses were performed using R (v3.1.3) and visualized using the ggplot2 package (v2.2.1; Wickham 2016).

\section{Acknowledgments}

We thank Angela Morley, Alan Ng, Monica Yu, and Hillary Winstanley for excellent care of zebrafish and facility maintenance. We thank Robert Gerlai, Yohaan Fernandes, Laura McDonald, Sarah Hutchinson, and Emanuela Pannia for helpful discussions. This work was supported by a grant from the Canadian Institute for Health Research to P.W.F. (FDN143227). J. W.K. was supported by a Human Frontiers Science Program longterm fellowship (LT000759/2014). P.W.F. and S.A.J. are senior fellows in the Canadian Institute for Advanced Research (CIFAR) programs in Child and Brain Development and Brain, Mind and Consciousness programs, respectively.

\section{References}

Abel T, Lattal KM. 2001. Molecular mechanisms of memory acquisition, consolidation and retrieval. Curr Opin Neurobiol 11: 180-187.

Agetsuma M, Aizawa H, Aoki T, Nakayama R, Takahoko M, Goto M, Sassa T, Amo R, Shiraki T, Kawakami K, et al. 2010. The habenula is crucial for experience-dependent modification of fear responses in zebrafish. Nat Neurosci 13: 1354-1356.

Ahrens MB, Li JM, Orger MB, Robson DN, Schier AF, Engert F, Portugues R. 2012. Brain-wide neuronal dynamics during motor adaptation in zebrafish. Nature 485: 471-477.

Aizenberg M, Schuman EM. 2011. Cerebellar-dependent learning in larval zebrafish. J Neurosci 31: 8708-8712.

Arthur D, Levin E. 2001. Spatial and non-spatial visual discrimination learning in zebrafish (Danio rerio). Anim Cogn 4: 125-131.

Blank M, Guerim LD, Cordeiro RF, Vianna MRMM. 2009. A one-trial inhibitory avoidance task to zebrafish: rapid acquisition of an NMDA-dependent long-term memory. Neurobiol Learn Mem 92: 529-534. 
Blaser RE, Vira DG. 2014. Experiments on learning in zebrafish (Danio rerio): a promising model of neurocognitive function. Neurosci Biobehav Rev 42: 224-231.

Branson K, Robie AA, Bender J, Perona P, Dickinson MH. 2009. High-throughput ethomics in large groups of Drosophila. Nat Methods 6: $451-457$.

Camp M, Norcross M, Whittle N, Feyder M, D'Hanis W, Yilmazer-Hanke D, Singewald N, Holmes A. 2009. Impaired Pavlovian fear extinction is a common phenotype across genetic lineages of the 129 inbred mouse strain. Genes Brain Behav 8: 744-752.

Clineschmidt BV, Martin GE, Bunting PR, Papp NL. 1982. Central sympathomimetic activity of (+)-5-methyl-10,11-dihydro-5H-dibenzo [a, d]cyclohepten-5,10-imine (MK-801), a substance with potent anticonvulsant, central sympathomimetic, and apparent anxiolytic properties. Drug Dev Res 2: 135-145.

Colwill RM, Raymond MP, Ferreira L, Escudero H. 2005. Visual discrimination learning in zebrafish (Danio rerio). Behav Processes 70 $19-31$.

Darland T, Dowling JE. 2001. Behavioral screening for cocaine sensitivity in mutagenized zebrafish. Proc Natl Acad Sci 98: 11691-11696.

Fanselow MS, Kim JJ, Yipp J, De Oca B. 1994. Differential effects of the Af-methyl-D-aspartate antagonist DL-2-amino-5-phosphonovalerate on acquisition of fear of auditory and contextual cues. Behav Neurosci 108: 235-240.

Fernandes YM, Rampersad M, Luchiari AC, Gerlai R. 2016. Associative learning in the multichamber tank: a new learning paradigm for zebrafish. Behav Brain Res 312: 279-284.

Frankland PW, Bontempi B. 2005. The organization of recent and remote memories. Nat Rev Neurosci 6: 119-130.

Frankland PW, Köhler S, Josselyn SA. 2013. Hippocampal neurogenesis and forgetting. Trends Neurosci 36: 497-503.

Gerlai R. 1998. Contextual learning and cue association in fear conditioning in mice: a strain comparison and a lesion study. Behav Brain Res 95: 191-203.

Gerlai R. 2010. High-throughput behavioral screens: the first step towards finding genes involved in vertebrate brain function using zebrafish. Molecules 15: 2609-2622.

Gerlai R. 2015. Zebrafish phenomics: behavioral screens and phenotyping of mutagenized fish. Curr Opin Behav Sci 2: 21-27.

Gorissen M, Manuel R, Pelgrim TNM, Mes W, de Wolf MJS, Zethof J, Flik G, van den Bos R. 2015. Differences in inhibitory avoidance, cortisol and brain gene expression in TL and AB zebrafish. Genes Brain Behav 14: $428-438$.

Grunwald DJ, Eisen JS. 2002. Headwaters of the zebrafish - emergence of a new model vertebrate. Nat Rev Genet 3: 717-724.

Guryev V, Koudijs MJ, Berezikov E, Johnson SL, Plasterk RHA, van Eeden FJM, Cuppen E. 2006. Genetic variation in the zebrafish. Genome Res 16: 491-497.

Hall D, Suboski MD. 1995. Visual and olfactory stimuli in learned release of alarm reactions by zebra danio fish (Brachydanio rerio). Neurobiol Learn Mem 63: 229-240.

Hisano Y, Sakuma T, Nakade S, Ohga R, Ota S, Okamoto H, Yamamoto T, Kawahara A, Hwang WY, Bedell VM, et al. 2015. Precise in-frame integration of exogenous DNA mediated by CRISPR/Cas9 system in zebrafish. Sci Rep 5: 8841.

Hohn C, Petrie-Hanson L. 2013. Evaluation of visible implant elastomer tags in zebrafish (Danio rerio). Biol Open 2: 1397-1401.

Houston FP, Stevenson GD, McNaughton BL, Barnes CA. 1999. Effects of age on the generalization and incubation of memory in the F344 rat. Learn Mem 6: 111-119.

Howe K, Clark MD, Torroja CF, Torrance J, Berthelot C, Muffato M, Collins JE, Humphray S, McLaren K, Matthews L, et al. 2013. The zebrafish reference genome sequence and its relationship to the human genome. Nature 496: 498-503.

Hwang WY, Fu Y, Reyon D, Maeder ML, Tsai SQ, Sander JD, Peterson RT, Yeh J-RJ, Joung JK. 2013. Efficient genome editing in zebrafish using a CRISPR-Cas system. Nat Biotechnol 31: 227-229.

Johansen JP, Cain CK, Ostroff LE, LeDoux JE. 2011. Molecular mechanisms of fear learning and memory. Cell 147: 509-524.

Kalueff AV, Gebhardt M, Stewart AM, Cachat JM, Brimmer M, Chawla JS, Craddock C, Kyzar EJ, Roth A, Landsman S, et al. 2013. Towards a comprehensive catalog of zebrafish behavior 1.0 and beyond. Zebrafish 10: $70-86$.

Kalueff AV, Stewart AM, Gerlai R. 2014. Zebrafish as an emerging model for studying complex brain disorders. Trends Pharmacol Sci 35: 63-75.
Leung LC, Mourrain P. 2016. Drug discovery: zebrafish uncover novel antipsychotics. Nat Chem Biol 12: 468-469.

Lieschke GJ, Currie PD. 2007. Animal models of human disease: zebrafish swim into view. Nat Rev Genet 8: 353-367.

MacRae CA, Peterson RT. 2015. Zebrafish as tools for drug discovery. Nat Rev Drug Discov 14: 721-731.

Manuel R, Gorissen M, Piza Roca C, Zethof J, van de Vis H, Flik G, Van Den Bos R. 2014. Inhibitory avoidance learning in zebrafish (Danio Rerio): effects of shock intensity and unraveling differences in task performance. Zebrafish 11: 341-352.

Maren S, Phan KL, Liberzon I. 2013. The contextual brain: implications for fear conditioning, extinction and psychopathology. Nat Rev Neurosci 14: 417-428.

Muto A, Orger MB, Wehman AM, Smear MC, Kay JN, Page-McCaw PS, Gahtan E, Xiao T, Nevin LM, Gosse NJ, et al. 2005. Forward genetic analysis of visual behavior in zebrafish. PLoS Genet 1: e66.

Owen EH, Logue SF, Rasmussen DL, Wehner JM. 1997. Assessment of learning by the Morris water task and fear conditioning in inbred mouse strains and F1 hybrids: implications of genetic background for single gene mutations and quantitative trait loci analyses. Neuroscience 80: 1087-1099.

Piato ÂL, Capiotti KM, Tamborski AR, Oses JP, Barcellos LJG, Bogo MR, Lara DR, Vianna MR, Bonan CD. 2011. Unpredictable chronic stress model in zebrafish (Danio rerio): behavioral and physiological responses. Prog Neuropsychopharmacol Biol Psychiatry 35: 561-567.

Roberts AC, Reichl J, Song MY, Dearinger AD, Moridzadeh N, Lu ED, Pearce K, Esdin J, Glanzman DL. 2011. Habituation of the C-start response in larval zebrafish exhibits several distinct phases and sensitivity to NMDA receptor blockade. PLoS One 6: e29132.

Rudy JW, O'Reilly RC. 1999. Contextual fear conditioning, conjunctive representations, pattern completion, and the hippocampus. Behav Neurosci 113: 867-880.

Schmid B, Haas C. 2013. Zebrafish as an animal model for neurodegeneration. J Neurochem 127: 461-470.

Sison M, Gerlai R. 2010. Associative learning in zebrafish (Danio rerio) in the plus maze. Behav Brain Res 207: 99-104.

Sison M, Gerlai R. 2011a. Associative learning performance is impaired in zebrafish (Danio rerio) by the NMDA-R antagonist MK-801. Neurobiol Learn Mem 96: 230-237.

Sison M, Gerlai R. 2011b. Behavioral performance altering effects of MK-801 in zebrafish (Danio rerio). Behav Brain Res 220: 331-337.

Stiedl O, Radulovic J, Lohmann R, Birkenfeld K, Palve M, Kammermeier J, Sananbenesi F, Spiess J. 1999. Strain and substrain differences in context- and tone-dependent fear conditioning of inbred mice. Behav Brain Res 104: 1-12.

Swain HA, Sigstad C, Scalzo FM. 2004. Effects of dizocilpine (MK-801) on circling behavior, swimming activity, and place preference in zebrafish (Danio rerio). Neurotoxicol Teratol 26: 725-729.

Trevarrow B, Robison B. 2004. Genetic backgrounds, standard lines, and husbandry of zebrafish. Methods Cell Biol, 77: 599-616.

Valente A, Huang K-H, Portugues R, Engert F. 2012. Ontogeny of classical and operant learning behaviors in zebrafish. Learn Mem 19: 170-177.

Varshney GK, Pei W, LaFave MC, Idol J, Xu L, Gallardo V, Carrington B, Bishop K, Jones M, Li M, et al. 2015. High-throughput gene targeting and phenotyping in zebrafish using CRISPR/Cas9. Genome Res 25: 1030-1042.

Vignet C, Bégout M-L, Péan S, Lyphout L, Leguay D, Cousin X. 2013. Systematic screening of behavioral responses in two zebrafish strains. Zebrafish 10: $365-375$.

Wickham H. 2016. ggplot2: elegant graphics for data analysis. Springer.

Wixted JT. 2004. The psychology and neuroscience of forgetting. Annu Rev Psychol 55: 235-269.

Wu J, Zou H, Strong JA, Yu J, Zhou X, Xie Q, Zhao G, Jin M, Yu L. 2005. Bimodal effects of MK-801 on locomotion and stereotypy in C57BL/6 mice. Psychopharmacology (Berl) 177: 256-263.

Xu X, Scott-Scheiern T, Kempker L, Simons K. 2007. Active avoidance conditioning in zebrafish (Danio rerio). Neurobiol Learn Mem 87: 72-77.

Yu L, Tucci V, Kishi S, Zhdanova IV. 2006. Cognitive aging in zebrafish. PLoS One 1: e14.

Zon LI, Peterson RT. 2005. In vivo drug discovery in the zebrafish. Nat Rev Drug Discov 4: 35-44.

Received April 3, 2017; accepted in revised form May 30, 2017. 


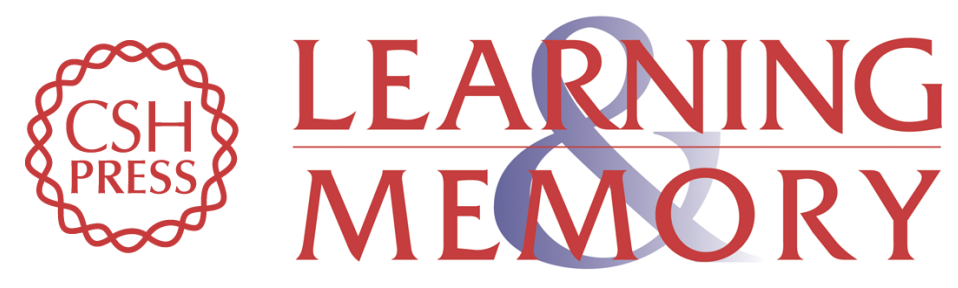

\section{Contextual fear conditioning in zebrafish}

Justin W. Kenney, Ian C. Scott, Sheena A. Josselyn, et al.

Learn. Mem. 2017, 24:

Access the most recent version at doi:10.1101//m.045690.117

References This article cites 56 articles, 7 of which can be accessed free at: http://learnmem.cshlp.org/content/24/10/516.full.html\#ref-list-1

Creative This article is distributed exclusively by Cold Spring Harbor Laboratory Press for the Commons first 12 months after the full-issue publication date (see License http://learnmem.cshlp.org/site/misc/terms.xhtml). After 12 months, it is available under a Creative Commons License (Attribution-NonCommercial 4.0 International), as described at http://creativecommons.org/licenses/by-nc/4.0/.

Email Alerting Receive free email alerts when new articles cite this article - sign up in the box at the Service top right corner of the article or click here. 